\section{BMJ Open Respiratory Research}

\title{
Characteristics of new adult users of mepolizumab with asthma in the USA
}

Ann Chen Wu, ${ }^{1}$ Pamela M McMahon, ${ }^{1}$ Emily Welch, ${ }^{1}$ Cheryl N McMahill-Walraven, ${ }^{2}$ Aziza Jamal-Allial, ${ }^{3}$ Mia Gallagher, ${ }^{1}$ Tancy Zhang, ${ }^{1}$ Christine Draper, ${ }^{1}$ Anne Marie Kline, ${ }^{2}$ Leslie Koerner, ${ }^{3}$ Jeffrey S Brown, ${ }^{1}$ Melissa K Van Dyke ${ }^{4}$

To cite: Wu AC, McMahon PM, Welch E, et al. Characteristics of new adult users of mepolizumab with asthma in the USA. BMJ Open Resp Res 2021;8:e001003. doi:10.1136/ bmjresp-2021-001003

Received 9 July 2021 Accepted 20 October 2021

\section{Check for updates}

(c) Author(s) (or their employer(s)) 2021. Re-use permitted under CC BY-NC. No commercial re-use. See rights and permissions. Published by BMJ.

${ }^{1}$ Department of Population Medicine, Harvard Medical School, Boston, Massachusetts, USA

${ }^{2}$ Aetna, Blue Bell,

Pennsylvania, USA

${ }^{3}$ HealthCore, Watertown,

Massachusetts, USA

${ }^{4}$ GlaxoSmithKline, Collegeville, Pennsylvania, USA

Correspondence to Dr Ann Chen Wu; Ann.Wu@childrens.harvard. edu

\section{ABSTRACT}

Background In the USA, over 25 million people have asthma; $5 \%-10 \%$ of cases are severe. Mepolizumab (Nucala) is an interleukin-5 antagonist monoclonal antibody; it was approved by the FDA in 2015 as add-on maintenance treatment of severe asthma for patients aged $\geq 12$ years with an eosinophilic phenotype.

Objectives (1) Describe baseline demographic and clinical characteristics of new US adult mepolizumab users 20152019, (2) describe asthma medication use in the 12 months preceding initiation of and concomitant with mepolizumab and (3) assess mepolizumab adherence, persistence and discontinuation patterns in 12 months postinitiation.

Methods We conducted a new-user observational cohort study using data from Aetna, a CVS Health Company, HealthCore (Anthem), Harvard Pilgrim Healthcare, and IBM MarketScan Research Databases. Curated administrative claims data in the FDA Sentinel System common data model format and publicly available Sentinel analytical tools were used to query the databases. We included adults who initiated mepolizumab in 2015-2019 with an asthma diagnosis in the preceding 12 months and no evidence of cystic fibrosis. We examined age, sex, comorbid conditions, asthma medication use and severe asthma exacerbations.

Results We identified 3496 adults (mean age 54.2 years, SD 12.5 years) who initiated mepolizumab. In the 12 months before mepolizumab initiation, $22 \%$ had received inhaled corticosteroids, $46 \%$ had inhaled corticosteroid/long-acting beta agonists, $72.6 \%$ had leukotriene antagonists, $38 \%$ had long-acting muscarinic antagonist, $18 \%$ had omalizumab, $<1 \%$ had reslizumab, dupilumab or benralizumab. In the previous 12 months, $70 \%$ had a diagnosis of allergic rhinitis, $32 \%$ had chronic obstructive pulmonary disease, $17 \%$ eosinophilia and $3 \%$ eosinophilic granulomatosis with polyangiitis. Further, $56 \%$ had an asthma-related ambulatory visit, $73 \% \geq 1$ course of oral corticosteroids lasting 3-27 days, $10 \%$ an asthma-related emergency department visit and $22 \%$ an asthma-related hospitalisation. In the 12 months following initiation, the mean proportion of days covered was $70 \%$, and reductions in the average mean dispensings of rescue oral corticosteriods (35\%) and omalizumab (61\%) were observed.

Conclusions Adults with asthma treated with mepolizumab had varying levels of healthcare utilisation and we observed evidence of mepolizumab use in patients without severe asthma.

\section{INTRODUCTION}

Globally, 350 million people have asthma, of which approximately 25.7 million live in the

\section{Key messages}

Adults with asthma treated with mepolizumab had varying asthma severity.

- Relatively few US adults with severe asthma are using mepolizumab; some received other biologics before initiating mepolizumab.

- This study evaluates baseline characteristics and treatment patterns among adult initiators of mepolizumab in a real-life population.

USA. ${ }^{12}$ Severe asthma-uncontrolled despite adherence with maximal therapy according to the Global Initiative for Asthma (GINA) occurs in approximately $5 \%-10 \%$ of the total asthma population. ${ }^{3}$ Asthma and severe asthma are heterogeneous. Although there are a range of possible classifications, one schema that can suggest potential treatment is to classify asthma as eosinophilic, neutrophilic, mixed or paucigranulocytic based on sputum samples. ${ }^{4}$ In adults with persistent asthma, high blood eosinophil counts have been found to be a risk factor for increased future asthma exacerbations.

Mepolizumab is an interleukin-5 (IL-5) antagonist monoclonal antibody approved by the US Food and Drug Administration (FDA) in 2015 for add-on maintenance treatment of severe asthma for patients aged $\geq 12$ years with an eosinophilic phenotype, its clinical efficacy evaluated in multiple randomised, doubleblind clinical trials. ${ }^{6}$ In the Dose Ranging Efficacy and Safety with Mepolizumab (DREAM) trial, clinically significant exacerbations were reduced by $48 \%$ in patient receiving mepolizumab compared with patients receiving placebo. ${ }^{7}$ Similarly, in the Mepolizumab as Adjunctive Therapy in Patients with Severe Asthma (MENSA) trial, clinically significant exacerbations were reduced by $53 \%$ among patients receiving mepolizumab compared with placebo. ${ }^{8}$ In the Steroid Reduction with Mepolizumab Study (SIRIUS) trial, the mean daily prednisone dose at weeks 20-24 
was higher among patients receiving placebo versus mepolizumab, resulting in a 2.4 times greater odds for oral glucocorticoid dose reduction from baseline in the mepolizumab group. ${ }^{9}$ Additionally, the mepolizumab add-on therapy on health-related quality of life and markers of asthma control in severe eosinophilic asthma (MUSCA) trial found that mepolizumab improved health-related quality of life in patients with severe eosinophilic asthma. ${ }^{10}$ Multiple clinical trials demonstrate the efficacy of mepolizumab for severe asthma; however, studies in large, real-life populations remain limited. ${ }^{11-13}$ A multisite study using a distributed data network offers the largest potential pool of new US users in real-life populations to evaluate treatment patterns of mepolizumab users. Overall, this study aimed to evaluate baseline characteristics and real-world treatment patterns among adult initiators of mepolizumab. Specific objectives were (1) describe baseline demographic and clinical characteristics of new US adult users of mepolizumab 2015-2019, (2) describe asthma medication use in the 12 months prior to initiation of and concomitant with mepolizumab and (3) assess adherence, persistence and discontinuation patterns of mepolizumab in 12 months postinitiation.

\section{METHODS}

\section{Patient and public involvement}

The development of the research question and outcome measures were informed based on anecdotal information from patients who have taken biologics. The outcome measures of asthma-related hospitalisations and emergency department (ED) visits have been previously demonstrated to be important to individuals with asthma. Given this study used deidentified aggregate data, no patients were involved in the design, recruitment and conduct of the study. Study participants are deidentified and results cannot be disseminated to them.

\section{Study design}

We conducted an observational cohort study using administrative claims data from three partners that participate in the FDA Sentinel System ${ }^{14}$ : Aetna, a CVS Health Company, HealthCore (Anthem), Harvard Pilgrim Health Care Institute (Harvard Pilgrim Health Care) and the IBM MarketScan Commercial Claims and Medicare Research databases. All data were formatted and curated using the FDA Sentinel common data model. ${ }^{15}$ Publicly available standardised analytical tools (https:// www.sentinelinitiative.org $/)^{16}$ were used to query each curated database separately using a distributed data network approach, and results were securely shared with the coordinating centre for aggregation.

\section{Study population}

The study population consisted of subjects aged $\geq 18$ years who initiated mepolizumab between 4 November 2015 (mepolizumab FDA approval date) and the most recent data available from each data partner (2018-2019). Figure 1 illustrates study population identification and provides details on coding for mepolizumab initiation. Mepolizumab use was identified via outpatient pharmacy

\section{Baseline period}

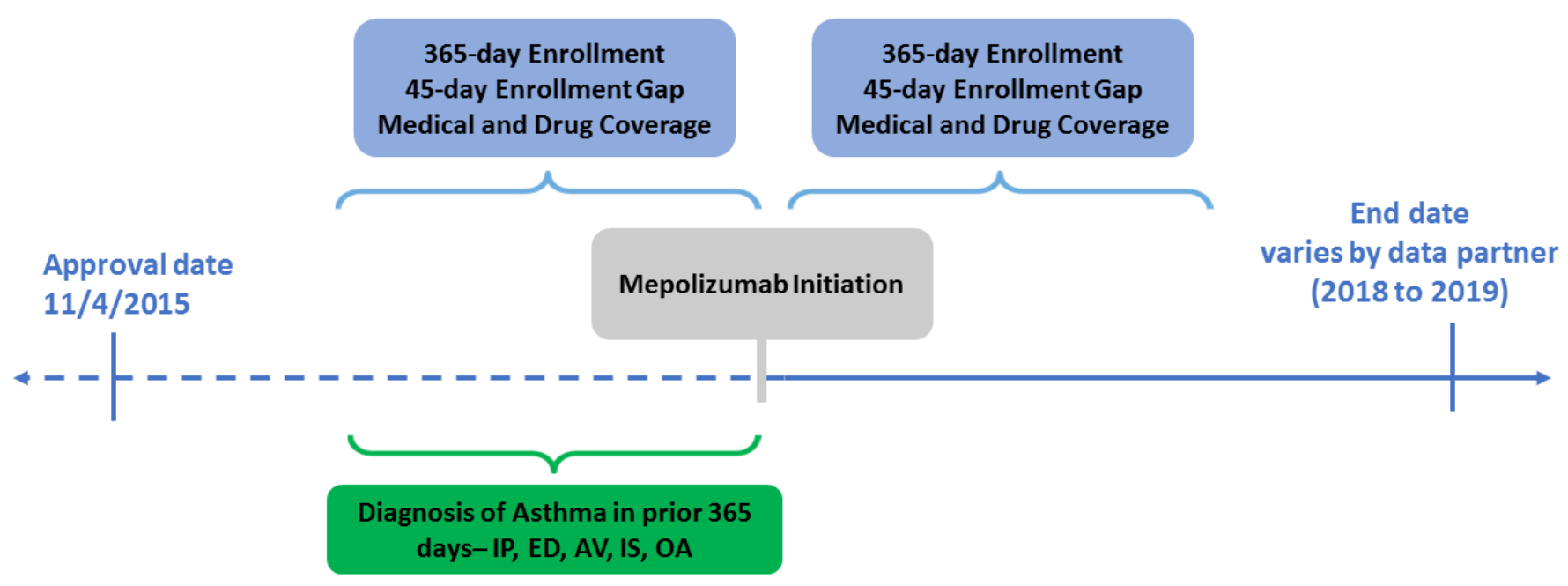

In subset with post-index period:

Adherence

Medication use

Figure 1 Identification and characterisation of new initiators of mepolizumab. Among adult patients identified with National Drug Codes (NDCs) in dispensing of medical claims files, $11 \%$ also had a procedure code in the medical claim file. COPD, chronic obstructive pulmonary disease. 
claims and medical claims using standards coding terminologies. Inclusion criteria were any diagnosis of asthma in the 12 months before mepolizumab initiation based on International Classification of Diseases (ICD), 9th Revision, Clinical Modification (ICD-9-CM 493.xx) or 10th Revision (ICD-10 J45.xx) during the study period. For evaluating medications used in the 12 months before mepolizumab initiation, we required 365 days of continuous health plan enrolment (allowing a 45-day enrolment gap) with medical and drug coverage in the period before the index date with no dispensing for mepolizumab (washout period). To assess adherence, persistence and discontinuation patterns of mepolizumab in the 12 months postinitiation, subjects were required to have 12 months of continuous enrolment postindex date (allowing a 45-day enrolment gap; subjects were followed until the earliest of the following events: health plan disenrolment, study end date, end of data availability or death). We excluded patients with any history of cystic fibrosis diagnosis claims (ICD-9-CM Code 277.0XX ICD-10 Code E84.XX) over the patients' entire enrolment history.

The primary exposure of interest was mepolizumab initiation. We defined mepolizumab initiation as the first mepolizumab dispensing record or procedure code after a 12-month wash-out period.

\section{Outcomes}

In subjects who initiated mepolizumab, had a diagnosis of asthma in the 365 days before mepolizumab initiation, and met enrolment criteria in the prior 365 days, we examined the following baseline demographic and clinical characteristics: age, sex, race/ethnicity, calendar year of initiation and Charlson/Elixhauser Combined Comorbidity Score defined using ICD-9-CM and ICD-10 codes. ${ }^{17}$ We further examined whether subjects had the following comorbid conditions, defined by ICD-9-CM and ICD-10 codes: allergic rhinitis, respiratory infections, sinusitis (acute/chronic), chronic obstructive pulmonary disease (COPD), nasal polyps, eosinophilia, rheumatoid arthritis, eosinophilic granulomatosis with polyangiitis, atopic dermatitis, chronic idiopathic urticaria and eosinophilic oesophagitis.

Based on GINA guidelines, ${ }^{18}$ subjects were classified with severe asthma if in the 90 days before mepolizumab initiation they received a medium-dose inhaled corticosteroid (ICS) plus long-acting beta agonists (LABA) in single or multiple devices (within 30 days of each other), systemic steroids (ie, $\geq 28$ days), anti-IgE treatment (eg, Xolair/omalizumab), tiotropium, anti-IL-5, high-dose ICS and leukotriene receptor antagonists (LTRA) (within 30 days of each other) or high-dose ICS.

A severe asthma exacerbation was defined as an asthma-related hospitalisation, asthma-related ED visit or need for oral corticosteroids of 3-27 days within 2 weeks (before and after) an outpatient asthma claim. Outpatient asthma-related visits and asthma diagnosis during hospitalisation in which asthma is the primary reason for the hospitalisation were also included as severe asthma exacerbations. As moderate exacerbations share a service location with scheduled mepolizumab administration visits, we excluded outpatient visits coded as mepolizumab administration. We studied the following medications 12 months before and after mepolizumab initiation: OCS, ICS, short-acting beta agonist, long-acting muscarinic antagonist (LAMA), LTRA, ICS/LABA, omalizumab, reslizumab, benralizumab and dupilumab. Unpaired, weighted t-tests were used to compare mean numbers of dispensings in the premepolizumab and postmepolizumab periods. Medications dispensed within 30 days of mepolizumab were considered concomitant.

To measure adherence, we calculated the proportion of days covered (PDC) by dividing the days supplied by 366 days, using an index date based on the first dispensing of mepolizumab. For the PDC calculation to be valid, we required 365 days of continuous enrolment (allowing a 45-day enrolment gap) after the index date and ended patients' follow-up on day 366. One dose of mepolizumab covers a 4-week period, so is equivalent to a 28-day supply. We defined cut-offs of PDC by deciles of a year (36.6 days) and estimated 95\% CIs. ${ }^{19}$ Research shows that a PDC $>75 \%$ is associated with decreased asthma exacerbations, ${ }^{20}$ and PDC for asthma controller medications is often low. ${ }^{21}$

We calculated the number of days between each mepolizumab refill. Early-stage persistence was defined as a second fill within 29-57 days (4-8 weeks) and a third fill within 29-169 days (4-24 weeks) of the first fill. We also assessed discontinuation by determining the number of subjects with no refill of mepolizumab within $3,6,9$

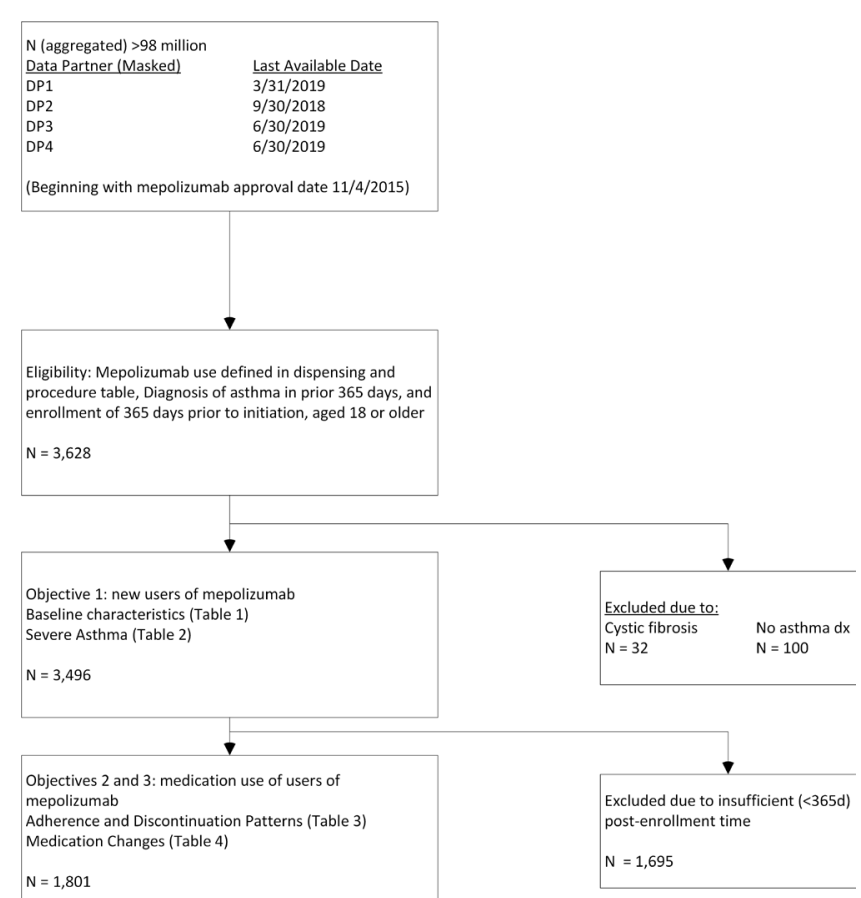

Figure 2 Study flow chart. 
Table 1 Demographic and clinical characteristics (in prior 12 months) of new users of mepolizumab ( $\mathrm{N}=3496)$

\begin{tabular}{|c|c|}
\hline Characteristic & Mean (N) \\
\hline Age in years (mean) & 54.2 (SD 12.5) \\
\hline \multicolumn{2}{|l|}{ Age category } \\
\hline $18-34$ years & $8 \%(262)$ \\
\hline $35-64$ years & $77 \%(2699)$ \\
\hline $65+$ years & $15 \%(535)$ \\
\hline Sex (female) & $63 \%(2193)$ \\
\hline \multicolumn{2}{|l|}{ Year } \\
\hline 2015 & $0.5 \%(17)$ \\
\hline 2016 & $24 \%(839)$ \\
\hline 2017 & $39 \%(1366)$ \\
\hline 2018 & $29 \%(1016)$ \\
\hline 2019 & $7 \%(258)$ \\
\hline Prior combined comorbidity score & 1.9 (SD 1.8) \\
\hline \multicolumn{2}{|l|}{ Comorbid illnesses in prior 12 months } \\
\hline Allergic rhinitis & $70 \%(2428)$ \\
\hline Atopic dermatitis & $3 \%(115)$ \\
\hline COPD & $32 \%(1117)$ \\
\hline Chronic idiopathic urticaria & $2 \%(56)$ \\
\hline Eosinophilia* & $17 \%(605)$ \\
\hline Eosinophilic oesophagitis & $2 \%(61)$ \\
\hline $\begin{array}{l}\text { Eosinophilic granulomatosis with } \\
\text { polyangiitis } \dagger\end{array}$ & $3 \%(118)$ \\
\hline Nasal polyps & $21 \%(727)$ \\
\hline Respiratory infections & $66 \%(2294)$ \\
\hline Rheumatoid arthritis & $3 \%(118)$ \\
\hline Sinusitis (acute/chronic) & $56 \%(1964)$ \\
\hline \multicolumn{2}{|c|}{ Asthma medications used in prior 12 months } \\
\hline Inhaled corticosteroids (ICS) & $22 \%(754)$ \\
\hline High-dose ICS and LTRA & $17 \%(582)$ \\
\hline ICS/LABA & $46 \%(1597)$ \\
\hline LTRA & $73 \%(2539)$ \\
\hline Long-acting muscarinic antagonist & $38 \%(1338)$ \\
\hline Oral corticosteroid (any days) & $91 \%(3164)$ \\
\hline Omalizumab & $18 \%(612)$ \\
\hline Reslizumab & $0.2 \%(8)$ \\
\hline Benralizumab & $1 \%(32)$ \\
\hline Dupilumab & $0.1 \%(3)$ \\
\hline Short-acting beta2-agonist & $84 \%$ (2928) \\
\hline
\end{tabular}

Severe asthma exacerbations in the prior 12 months (combined metric) $\ddagger$

$\begin{array}{ll}\text { None } & 14 \%(489) \\ 1 & 18 \%(646) \\ 2 \text { or more } & 68 \%(1136)\end{array}$

Hospitalisation with asthma as primary diagnosis

\begin{tabular}{|c|c|}
\hline Characteristic & Mean (N) \\
\hline None & $93 \%(3236)$ \\
\hline 1 & $6 \%(196)$ \\
\hline 2 or more & $2 \%(3433)$ \\
\hline \multicolumn{2}{|c|}{ Asthma-related hospitalisation } \\
\hline None & $85 \%(2978)$ \\
\hline 1 & $10 \%(359)$ \\
\hline 2 or more & $5 \%(3338)$ \\
\hline \multicolumn{2}{|c|}{ Asthma-related outpatient visit } \\
\hline None & $44 \%(1539)$ \\
\hline 1 & $22 \%(757)$ \\
\hline 2 or more & $34 \%(2297)$ \\
\hline \multicolumn{2}{|c|}{ Asthma-related ED visit } \\
\hline None & $90 \%(3153)$ \\
\hline 1 & $7 \%(248)$ \\
\hline 2 or more & $3 \%(3402)$ \\
\hline \multicolumn{2}{|c|}{$\begin{array}{l}\text { Exacerbation requiring oral corticosteroid (OCS) (days } \\
\text { supply between } 3 \text { and 27) }\end{array}$} \\
\hline None & $27 \%$ (959) \\
\hline 1 & $20 \%(693)$ \\
\hline 2 or more & $53 \%(1653)$ \\
\hline
\end{tabular}

*Overlap with other categories was possible.

†Mepolizumab dose for was not available.

$\ddagger$ Overall combined metric was defined as any of the individual exacerbations below.

COPD, chronic obstructive pulmonary disease; ED, emergency department; LABA, long-acting beta agonists; LTRA, leukotriene receptor antagonists.

and 12 months of mepolizumab initiation. Further, we examined gaps (number of days) between mepolizumab dispensings. We also calculated the time to discontinuation allowing a 28-day gap between dispensings.

\section{RESULTS}

From 4 November 2015 to 30 June 2019, we identified 3628 subjects with mepolizumab initiation and the required enrolment criteria (figure 2). Subjects without an asthma diagnosis in the prior 12 months $(\mathrm{n}=100)$ and with a cystic fibrosis diagnosis $(\mathrm{n}=32)$ were excluded, leaving 3496 adults: mean age 54.2 years, and $63 \%$ female. Race was unknown for most adults $(96 \%)$ and not included in table 1 . When the 12-month follow-up period was required (to assess medications used in the 12 months before and after initiation), our final sample size was 1801 (figure 2).

Descriptive data including baseline and clinical characteristics are provided in table 1 . In the 12 months before mepolizumab initiation, 22\% had received ICS, $46 \%$ ICS/LABA, 72.6\% leukotriene antagonists, 38\% LAMA, $18 \%$ omalizumab, $<1 \%$ reslizumab, $<1 \%$ dupilumab and $<1 \%$ benralizumab. In the previous 12 months, $70 \%$ 
Table 2 Controller medications for asthma dispensed in 12 months prior to mepolizumab

\begin{tabular}{lclc}
\hline & \multicolumn{2}{c}{ Medication use } & \\
\cline { 2 - 4 } & No dispensings & One or more dispensings & Two or more dispensings \\
\hline Severe asthma* & $45 \%(1561)$ & $55 \%(1935)$ & $14 \%(500)$ \\
\hline ICS alone & & & \\
\hline Low-dose ICS & $87 \%(3034)$ & $13 \%(462)$ & $5 \%(173)$ \\
\hline Medium-high ICS* & $78 \%(2712)$ & $22 \%(784)$ & $7 \%(243)$ \\
\hline High-dose inhaled corticosteroids (ICS)* & $87 \%(3053)$ & $13 \%(443)$ & $5 \%(161)$ \\
\hline ICS/LABA & & & $14 \%(490)$ \\
\hline Low-dose ICS/LABA & $66 \%(2292)$ & $34 \%(1,204)$ & $6 \%(211)$ \\
\hline Medium-high dose ICS/LABA* & $84 \%(2954)$ & $16 \%(542)$ & $20 \%(714)$ \\
\hline ICS/LABA (any dose) & $51 \%(1800)$ & $49 \%(1696)$ & $2 \%(76)$ \\
\hline ICS/LTRA & & & $2 \%(67)$ \\
\hline Low-dose ICS/LTRA & $92 \%(3225)$ & $8 \%(271)$ & $9 \%(303)$ \\
\hline High-dose ICS/LTRA* & $93 \%(3234)$ & $7 \%(262)$ & $0 \%(3)$ \\
\hline Biologics & & & $0 \%(7)$ \\
\hline Omalizumab* & & $12 \%(303)$ & $0 \%(2)$ \\
\hline Reslizumab* & $88 \%(3079)$ & $13 \%(470)$ \\
\hline Benralizumab* & $100 \%(3491)$ & $0 \%(3)$ & $6 \%(218)$ \\
\hline Dupilumab* & $99 \%(3478)$ & $1 \%(7)$ & \\
\hline Other controller medications & $100 \%(3493)$ & $0 \%(2)$ & \\
\hline Oral corticosteroids (days supply $\geq 28$ days) $^{*}$ & $72 \%(2525)$ & $28 \%(971)$ & \\
\hline Tiotropium & $77 \%(2680)$ & $23 \%(816)$ & \\
\hline
\end{tabular}

$\mathrm{N}=3496$.

*Medications included in definition of severe asthma.

ICS, inhaled corticosteroids; LABA, long-acting beta agonists; LTRA, leukotriene receptor antagonists.

had a diagnosis of allergic rhinitis, $32 \%$ had COPD, $17 \%$ eosinophilia and $3 \%$ eosinophilic granulomatosis with polyangiitis.

Table 2 also provides results on controller medications used, including medications that indicate severe asthma per GINA guidelines. ${ }^{18}$ In the 12 months prior to initiating mepolizumab, $55 \%$ of subjects had at least one dispensing of a medication indicating severe asthma (either medium or high dose ICS with LABA separately or as a combination product, $\geq 28$ days of systemic steroids, high-dose ICS with LTRA, omalizumab, dupilumab, tiotropium or an anti-IL-5 therapy, including reslizumab or benralizumab). Additional medications used include short courses of OCS (3-27 days, used by $55 \%$ of subjects), or any dose ICS/LABA (used by $49 \%$ ). Further, 28\% had at least a 28-day supply of systemic steroids. The most common category with minimum two dispensings was OCS (3-27 days), observed in 27\% of adults. Omalizumab was dispensed at least once to $12 \%$ of mepolizumab users. In the 12 months prior to initiating mepolizumab, most subjects had the combination of at least one outpatient asthma diagnosis and 3-27 days of oral corticosteroids supplied; most had an ambulatory asthma-related event, but few had any asthma-related ED events or inpatient stays. In our secondary analysis where we excluded the $46 \%$ of subjects with a COPD diagnosis at any time during the study period, medication use in the previous 12 months remained similar-23\% had received medium-high dose ICS, $67 \%$ received any dose of ICS/LABA and $35 \%$ took oral steroids of 28 days or more.

Results on adherence to mepolizumab, including PDC and discontinuation patterns, are provided in table 3 . The mean PDC was $70 \%$ in adults, with the median skewing higher $(80.0 \%)$. Few adults $(5.3 \%)$ had a $\mathrm{PDC}<10 \%$. Using an adherence definition of $\geq 70 \%$ PDC, we observed that $61.5 \%$ (95\% CI $60.25 \%$ to $62.75 \%$ ) of patients were adherent. Early-stage persistence was $65.3 \%$. The patterns of discontinuation over time showed little change after 6 months. The median time to discontinuation was 28 days, with a mean value skewed higher (68 days). When a 28-day gap was allowed, the median time to discontinuation was 271 days, with a mean value of 233 days.

Table 4 provides weighted averages of numbers of dispensings of each medication use category for the 12-month periods before and after mepolizumab initiation. We observed about one fewer dispensing over 12 months in oral corticosteroids (3-27 days; $\mathrm{p}<0.0001)$ and omalizumab $(\mathrm{p}<0.0001)$, an increase in LAMA use 


\begin{tabular}{|ll}
\hline Table $3 \quad$ Adherence to mepolizumab & \\
\hline Descriptive statistics & \\
\hline Proportion of days covered (PDC, (mean)) & 0.7 \\
\hline PDC (median) & 0.8 \\
\hline No/per cent patients by PDC category & $\%(n)$ \\
\hline PDC $\geq 25 \%$ & $91(1643)$ \\
\hline PDC $\geq 50 \%$ & $77(1377)$ \\
\hline PDC $\geq 75 \%$ & $62(1107)$ \\
\hline PDC $=100 \%$ & $2(38)$ \\
\hline No/per cent patients & $\% \mathrm{n}$ \\
\hline Early-stage persistence & $65(1176)$ \\
\hline Discontinuation by catgeory & $\%(\mathrm{n})$ \\
\hline No refill within 3 months & $11(202)$ \\
\hline No refill within 6 months & $7(120)$ \\
\hline No refill within 9 months & $6(106)$ \\
\hline No refill within 12 months & $6(99)$ \\
\hline Time to discontinuation & No of days \\
\hline Mean & 68 \\
\hline Median & 28 \\
\hline With 28-day allowable gap (mean) & 233 \\
\hline With 28-day allowable gap (median) & 271 \\
\hline
\end{tabular}

$\mathrm{N}=1801$.

( $p<0.0001$ ), and increases (from very rare to rare) in dupilumab $(\mathrm{p}<0.0001)$, benralizumab $(\mathrm{p}<0.0001)$ and reslizumab $(\mathrm{p}=0.017)$.

\section{DISCUSSION}

Our study has three key findings. First, mepolizumab is being used in US adults with asthma with a range of comorbid illnesses and prior medication use. Second, many individuals receiving mepolizumab do not have severe asthma. Third, adherence to mepolizumab is relatively high compared with other controller asthma medications that are not biologics.

Use of mepolizumab has grown since its FDA approval in 2015, yet few studies have examined mepolizumab use in real-life populations. Of adults receiving mepolizumab, patients in our study received a range of controller medications including ICS, ICS/LABA, LTRAs, LAMAs and other biologics, such as omalizumab. Prescription fills of oral corticosteroids as a controller medication and omalizumab were decreased in individuals prescribed mepolizumab. The most common comorbid illnesses were allergic rhinitis, sinusitis and respiratory tract infections-similar to findings in an effectiveness study by Llanos et al, of 346 subjects with private health insurance who initiated mepolizumab. ${ }^{11}$ Furthermore, $84 \%$ of patients in the Llanos et al cohort had experienced an asthma exacerbation-an outpatient or ED visit with an asthma diagnosis and at least one prescription of systemic steroids within 5 days of the encounter-in the prior year. ${ }^{11}$ We also found that $86 \%$ of subjects had at least one asthma-related hospitalisation, ED visit, outpatient visit or exacerbation requiring OCS, although we did not define an asthma exacerbation as Llanos et al, did. Our results demonstrate to clinicians, payers, and policymakers that mepolizumab is being used for patients with asthma with varying types of controller medication use in the prior 12 months.

Although mepolizumab is recommended only for severe asthma, only $55 \%$ of subjects receiving mepolizumab met severe asthma criteria. These findings are significant as clinicians may not be following guidelines when initiating mepolizumab treatment. According to GINA guidelines, ${ }^{18}$ subjects are classified as having severe asthma if they were treated with high dose ICS, medium or high-dose ICS/LABA, high-dose ICS/LTRA, systemic steroids, omalizumab, tiotropium or anti-IL-5. The study by Llanos et al reported that $14 \%$ of subjects received a medium dose ICS and 49\% a high-dose ICS in the 12 months prior to initiating mepolizumab. ${ }^{11}$ While a higher percentage of subjects in the Llanos $e t$ al study received a medium or high dose ICS (64\%) than our study (55\%), possible explanations of the discrepancy include that the Llanos et al study was significantly smaller at 346 subjects, roughly one-fifth of our sample, and they excluded subjects who filled omalizumab, reslizumab, benralizumab or dupilumab whereas we do not. ${ }^{11}$ Studies of new initiators of omalizumab, which was FDA-approved in 2003 and is recommended for patients with severe allergic asthma that remains uncontrolled despite highdose ICS/LABAs, demonstrated that many omalizumab new users had good asthma control and insufficient use of ICS or ICS/LABA in the 12 months prior to qualify them for omalizumab. ${ }^{22}{ }^{23}$ More specifically, Jeffery et al reported that almost half $(49 \%)$ of omalizumab initiators in their analysis had very low adherence to ICS or ICS/LABA with a medication possession ratio $(\leq 0.50)$, suggesting that these patients could have been better controlled on ICS or ICS/LABA and did not require omalizumab. ${ }^{24}$ Similarly, Verhamme et al found that in Belgium, only $24 \%$ of patients receiving omalizumab met eligibility criteria as the majority of omalizumab initiators are nonadherent to ICSs and/or ICS/LABAs. ${ }^{23}$ The findings that omalizumab and mepolizumab are started in patients who may not have exhausted management with other controller medications may be related to the low overall use of asthma biologics, such that providers do not have a lot of experience with prescribing. ${ }^{25}$ Nevertheless, many insurers have restrictions in place to prevent most patients from receiving mepolizumab unless they have severe asthma.

Inselman et al reported that $65 \%$ of clinicians prescribed only one type of biologic and concluded that clinicians may need additional logistical support to deliver asthma biologics to patients to remain consistent with guidelines. ${ }^{25}$ Moreover, some investigators have suggested that biologics such as omalizumab may offer a good alternative for patients with poor adherence to 
Table 4 Change in medication use in 12 months prior to versus postinitiation of mepolizumab

\begin{tabular}{|c|c|c|c|c|c|}
\hline Medication & $\begin{array}{l}\text { Premepolizumab } \\
\text { mean-weighted } \\
\text { average dispensings }\end{array}$ & Pre-SD & $\begin{array}{l}\text { Postmepolizumab } \\
\text { mean-weighted } \\
\text { average dispensings }\end{array}$ & Post-SD & $\begin{array}{l}\text { Per cent reduction } \\
\text { (negative indicates } \\
\text { increase) }\end{array}$ \\
\hline \multicolumn{6}{|l|}{ Single controller medication } \\
\hline Low ICS or medium-dose ICS & 0.74 & 3 & 0.71 & 3.3 & 5 \\
\hline High-dose ICS & 0.61 & 2.8 & 0.67 & 3.4 & -10 \\
\hline LAMA & 1.3 & 3.3 & 1.6 & 4.1 & -22 \\
\hline LTRA & 3.3 & 3.8 & 3.5 & 4.3 & -4 \\
\hline Oral corticosteroids (28+ days) & 1.5 & 3.3 & 1.6 & 3.7 & -5 \\
\hline \multicolumn{6}{|l|}{ Combination } \\
\hline ICS/LABA (any dose) & 2.7 & 3.3 & 2.8 & 3.8 & -4 \\
\hline Low-dose ICS/LABA & 1.8 & 3.1 & 2 & 3.7 & -9 \\
\hline Medium-dose or high-dose ICS/ LABA & 0.91 & 3.3 & 0.86 & 3.8 & 5 \\
\hline Low-dose or medium-dose ICS/ LTRA & 0.34 & 2.2 & 0.33 & 2.6 & 3 \\
\hline \multicolumn{6}{|l|}{ ICS/LTRA } \\
\hline High-dose ICS/ LTRA & 0.29 & 2.1 & 0.31 & 2.6 & -7 \\
\hline \multicolumn{6}{|l|}{ Biologics } \\
\hline Omalizumab & 1.4 & 6.6 & 0.54 & 6.4 & 61 \\
\hline Reslizumab & 0.001 & 0.71 & 0.028 & 3.6 & -4992 \\
\hline Benralizumab & 0.001 & 1.4 & 0.075 & 2.5 & -6650 \\
\hline Dupilumab & 0.004 & 2.1 & 0.036 & 3.7 & -712 \\
\hline \multicolumn{6}{|l|}{ Rescue } \\
\hline Oral corticosteroids (3-27 days) & 3.2 & 3.5 & 2.1 & 2.8 & $35 \%$ \\
\hline SABA & 3.8 & 4.6 & 3.6 & 4.9 & $6 \%$ \\
\hline
\end{tabular}

Paired measurements were not available due to aggregated nature of data.

ICS, inhaled corticosteroids; LABA, long-acting beta agonists; LAMA, long-acting muscarinic antagonist; LTRA, leukotriene receptor antagonists; SABA, short-acting beta agonist.

inhaled controller medications whose adherence does not improve with interventions. ${ }^{26}$ Thus, it is plausible that some clinicians choose mepolizumab for patients nonadherent to inhaled controller medications with the hope that overall medication adherence will increase even though these patients do not meet criteria for severe asthma. Nevertheless, one reason for requiring strict criteria for prescribing biologics is their high costs; most cost-effectiveness studies of omalizumab and mepolizumab recommend targeting therapy to select populations in order to improve value. ${ }^{27}$ As more biologics become available-four became approved after 2015it is particularly important for clinicians to understand which patients should receive biologics. While patients receiving mepolizumab may not have evidence of severe asthma as defined in the study, many patients with severe asthma do not receive biologics. ${ }^{24}{ }^{28}$ Supporting the findings from other studies, our study suggests that clinicians may need support in choosing which patients should initiate mepolizumab for asthma.

Adherence to mepolizumab in the 12 months after initiation was high with the mean PDC $70 \%$. Adherence to controller medications for asthma is critical for preventing asthma-related exacerbations, which cost more than US $\$ 80$ billion annually when accounting for medical costs, asthma-related mortality and losses due to missed work and school days. ${ }^{29}$ Nevertheless, adherence to controller medications for asthma is poor. ${ }^{21}$ One benefit of mepolizumab is its dosing, once every 4 weeks, perhaps thus easier to be adherent to than inhalers that need daily administration. ${ }^{30}$ Moreover, studies have demonstrated that a 4-week dosing of omalizumab results in improved adherence compared with a 2-week dosing. ${ }^{31}$ No studies, to our knowledge, have examined adherence to mepolizumab, but our findings of relatively high adherence are reassuring. Studies of adherence to omalizumab report high adherence with Campisi et al, finding that $88 \%$ of patients receiving omalizumab for less than 2 years had good adherence: patients in that study missed $<10 \%$ of scheduled doses. ${ }^{32}$ Adherence increased to $100 \%$ for patients taking omalizumab for more than 4 years. ${ }^{32}$ Research by Li et al found a mean PDC of $74 \%$ for omalizumab with $61 \%$ of patients having a $\mathrm{PDC} \geq 80 \%$, similar to our findings for mepolizumab. ${ }^{33}$ The adherence that we saw in our study is with mepolizumab administered in hospitals and clinics; with the approval of the Nucala Autoinjector that allows at-home administration of mepolizumab,${ }^{34}$ adherence may increase. 
The fact that, to our knowledge, our study is the largest real-life study of mepolizumab initiators is a noteworthy strength. Additionally, by having access to insurer claims data, we have a more accurate representation of medications received by patients as opposed to relying on prescription data. The availability of claims data allows us an accurate measure of severe asthma, defined based on medications filled. Despite the strengths of this study, several caveats deserve mention. These results are limited to health insurance administration claims and may not be generalisable to uninsured or other populations. Furthermore, exposure is inferred from claims in this study, and days of supply information from outpatient pharmacy claims was used to approximate on-treatment time for individual asthma patients exposed to mepolizumab and other medications for asthma. We were unable to share individual level data outside each health system which prevented our use of paired statistical tests for comparisons. We allowed for gaps in enrolment but otherwise required full 12 months of premepolizumab and postmepolizumab claims. Additionally, for mepolizumab new users, eosinophil laboratory values may be useful in evaluating whether therapy has been prescribed appropriately to patients with higher eosinophil values; however, laboratory information, including dosing information, is not available in these claims data. The reporting of eosinophilia in this manuscript report refers to an ICD-9 or ICD-10 diagnosis of eosinophilia. Moreover, some may believe that our inclusion of subjects with COPD who also had asthma in our main analysis could have contributed to the low percentage of subjects with severe asthma. Nevertheless, even after excluding patients with COPD, the proportion of subjects who met criteria for severe asthma was unchanged.

In summary, mepolizumab is being used increasingly in adults with asthma. Adults with asthma had varying levels of healthcare utilisation and comorbid diagnoses in the 12 months prior to initiating mepolizumab, and many new initiators of mepolizumab did not meet criteria for severe asthma.

Contributors AW is responsible for the overall content as guarantor. AW accepted full responsibility for the finished work and/or the conduct of the study, had access to the data and controlled the decision to publish. PM contributed to the planning, conduct and reporting of the work described in the article. EW contributed to the planning, conduct and reporting of the work described in the article. CNM-W contributed to the planning, conduct and reporting of the work described in the article. AJ-A contributed to the planning, conduct and reporting of the work described in the article. MG contributed to the planning and conduct of the work described in the article. TZ contributed to the planning, conduct and reporting of the work described in the article. CD contributed to the conduct and reporting of the work described in the article. AMK contributed to the planning, conduct and reporting of the work described in the article. LK contributed to the planning, conduct and reporting of the work described in the article. JSB contributed to the planning, conduct and reporting of the work described in the article. MKVD contributed to the planning, conduct and reporting of the work described in the article.

Funding This study was funded by GSK (PRJ2757).

Competing interests None declared.

Patient and public involvement Patients and/or the public were not involved in the design, or conduct, or reporting, or dissemination plans of this research.
Patient consent for publication Not applicable.

Ethics approval The Harvard Pilgrim Health Care IRB has determined this project does not meet the definition of human subject research under the purview of the IRB according to federal regulations. IRB REFERENCE \#: 1360801.

Provenance and peer review Not commissioned; internally peer reviewed.

Data availability statement Data are available on reasonable request.

Open access This is an open access article distributed in accordance with the Creative Commons Attribution Non Commercial (CC BY-NC 4.0) license, which permits others to distribute, remix, adapt, build upon this work non-commercially, and license their derivative works on different terms, provided the original work is properly cited, appropriate credit is given, any changes made indicated, and the use is non-commercial. See: http://creativecommons.org/licenses/by-nc/4.0/.

\section{REFERENCES}

1 Akinbami LJ, Moorman JE, Bailey C, et al. Trends in asthma prevalence, health care use, and mortality in the United States, 2001-2010. NCHS Data Brief 2012:1-8.

2 GBD 2015 Disease and Injury Incidence and Prevalence Collaborators. Global, regional, and national incidence, prevalence, and years lived with disability for 310 diseases and injuries, 19902015: a systematic analysis for the global burden of disease study 2015. Lancet 2016;388:1545-602.

3 Chung KF, Wenzel SE, Brozek JL, et al. International ERS/ATS guidelines on definition, evaluation and treatment of severe asthma. Eur Respir J 2014;43:343-73.

4 Anderson GP. Endotyping asthma: new insights into key pathogenic mechanisms in a complex, heterogeneous disease. Lancet 2008;372:1107-19.

5 Zeiger RS, Schatz M, Li Q, et al. High blood eosinophil count is a risk factor for future asthma exacerbations in adult persistent asthma. J Allergy Clin Immunol Pract 2014;2:741-50.

6 Fala L, Nucala FL. Nucala (mepolizumab): first IL-5 antagonist monoclonal antibody FDA approved for maintenance treatment of patients with severe asthma. Am Health Drug Benefits 2016;9:106-10.

7 Pavord ID, Korn S, Howarth P, et al. Mepolizumab for severe eosinophilic asthma (DREAM): a multicentre, double-blind, placebocontrolled trial. Lancet 2012;380:651-9.

8 Ortega HG, Liu MC, Pavord ID, et al. Mepolizumab treatment in patients with severe eosinophilic asthma. $N$ Engl $\mathrm{J} \mathrm{Med}$ 2014;371:1198-207.

9 Bel EH, Wenzel SE, Thompson PJ, et al. Oral glucocorticoid-sparing effect of mepolizumab in eosinophilic asthma. N Engl J Med 2014;371:1189-97.

10 Chupp GL, Bradford ES, Albers FC, et al. Efficacy of mepolizumab add-on therapy on health-related quality of life and markers of asthma control in severe eosinophilic asthma (Musca): a randomised, double-blind, placebo-controlled, parallel-group, multicentre, phase 3B trial. Lancet Respir Med 2017;5:390-400.

11 Llanos J-P, Ortega H, Bogart M, et al. Real-World effectiveness of mepolizumab in patients with severe asthma: an examination of exacerbations and costs. J Asthma Allergy 2020;13:77-87.

12 van Toor JJ, van der Mark SC, Kappen JH, et al. Mepolizumab addon therapy in a real world cohort of patients with severe eosinophilic asthma: response rate, effectiveness, and safety. J Asthma 2021;58:651-8.

13 Harrison T, Canonica GW, Chupp G, et al. Real-World mepolizumab in the prospective severe asthma REALITI-A study: initial analysis. Eur Respir J 2020;56:2000151.

14 Platt R, Brown JS, Robb M, et al. The FDA sentinel initiative - an evolving national resource. $N$ Engl J Med Overseas Ed 2018;379:2091-3.

15 Curtis LH, Weiner MG, Boudreau DM, et al. Design considerations, architecture, and use of the Mini-Sentinel distributed data system. Pharmacoepidemiol Drug Saf 2012;21 Suppl 1:23-31.

16 Sentinel Initiative. Routine Querying tools. Available: https://www. sentinelinitiative.org/sentinel/surveillance-tools/routine-queryingtools [Accessed 29 Apr 2020].

17 Gagne JJ, Glynn RJ, Avorn J, et al. A combined comorbidity score predicted mortality in elderly patients better than existing scores. $J$ Clin Epidemiol 2011;64:749-59.

18 Global Initiative for Asthma. Global strategy for asthma management and prevention, 2018. Available: www.ginasthma.org [Accessed 18 Mar 2019].

19 Ausvet. Sample size to estimate a proportion with specified precision, 2019. Available: http://epitools.ausvet.com.au/content. 
php? page $=1$ Proportion\&Proportion $=0.3 \&$ Precision $=0.05 \&$ Conf $=0$. 95\&Population $=$ [Accessed 05 Jun 2019].

20 Williams LK, Pladevall M, Xi H, et al. Relationship between adherence to inhaled corticosteroids and poor outcomes among adults with asthma. J Allergy Clin Immunol 2004;114:1288-93.

21 Wu AC, Butler MG, Li L, et al. Primary adherence to controller medications for asthma is poor. Ann Am Thorac Soc 2015;12:161-6.

22 Ke X, Kavati A, Wertz D, et al. Real-World clinical characteristics, treatment patterns, and exacerbations in US patients with asthma newly treated with omalizumab. Clin Ther 2018;40:1140-58.

23 Verhamme KMC, Lucet C, Van Meerhaeghe A, et al. Real-life effectiveness of omalizumab in difficult-to-treat versus severe asthma: a national cohort study in Belgium. ERJ Open Res 2019;5:00253-2018.

24 Jeffery MM, Shah ND, Karaca-Mandic P, et al. Trends in omalizumab utilization for asthma: evidence of suboptimal patient selection. $J$ Allergy Clin Immunol Pract 2018;6:1568-77.

25 Inselman JW, Jeffery MM, Maddux JT, et al. Trends and disparities in asthma biologic use in the United States. J Allergy Clin Immunol Pract 2020;8:549-54.

26 Hendeles L, Khan YR, Shuster JJ, et al. Omalizumab therapy for asthma patients with poor adherence to inhaled corticosteroid therapy. Ann Allergy Asthma Immunol 2015;114:58-62.
27 McQueen RB, Sheehan DN, Whittington MD, et al. CostEffectiveness of biological asthma treatments: a systematic review and recommendations for future economic evaluations. Pharmacoeconomics 2018;36:957-71.

28 Marques Mello L, Viana KP, Moraes Dos Santos F, et al. Severe asthma and eligibility for biologics in a Brazilian cohort. J Asthma 2021;58:958-66.

29 AAFA. Asthma facts. Available: https://www.aafa.org/asthma-facts/ [Accessed 14 Apr 2020].

30 Broder MS, Chang EY, Ory C, et al. Adherence and persistence with omalizumab and fluticasone/salmeterol within a managed care population. Allergy Asthma Proc 2009;30:148-57.

31 Janson SL, Solari PG, Trzaskoma B, et al. Omalizumab adherence in an observational study of patients with moderate to severe allergic asthma. Ann Allergy Asthma Immunol 2015;114:516-21.

32 Campisi R, Crimi C, Intravaia R, et al. Adherence to omalizumab: A multicenter "real-world" study. World Allergy Organ J 2020;13:100103.

33 Li P, Kavati A, Puckett JT, et al. Omalizumab treatment patterns among patients with asthma in the US Medicare population. $J$ Allergy Clin Immunol Pract 2020;8:507-15.

34 NUCALA (mepolizumab). NUCALA autoinjector. Available: https:// www.nucala.com/severe-asthma/about-nucala/nucala-autoinjector/ [Accessed 14 Apr 2020]. 